\title{
Exploring Antimicrobial Resistance in Agents Causing Urinary Tract Infections at a Tertiary Care Hospital in a Developing Country
}

Zuhair Ali Rizvi ${ }^{1}$, Ali Murad Jamal ${ }^{1}$, Ali Hassan Malik ${ }^{2}$, Syed Muhammad Jawad Zaidi ${ }^{1}$, Naimat Ullah Abdul Rahim $^{2}$, Daneyal Arshad ${ }^{2}$

1. Surgery, Rawalpindi Medical University, Rawalpindi, PAK 2. Urology, Rawalpindi Medical University, Rawalpindi, PAK

Corresponding author: Syed Muhammad Jawad Zaidi, mjawad927@gmail.com

\section{Abstract}

\section{Background and objective}

Urinary tract infections (UTIs) are usually treated with empirical therapy by physicians based on previous knowledge of the predictability of causative agents and their antimicrobial susceptibilities. The objective of this study was to determine the frequency of various pathogens causing UTIs and their antimicrobial resistance profile in patients presenting to the outpatient department (OPD) of a tertiary care hospital.

\section{Materials and methods}

This descriptive cross-sectional study was conducted in the urology OPD of a tertiary care hospital in Pakistan. The study was conducted over a period of six months, and it included 1,000 patients (of ages 12 years or above) who were clinically suspected for UTIs. Patients with comorbidities and immunocompromised patients were excluded from the study. Recipients of corticosteroid therapy or those with a history of intake of broad-spectrum antibiotics in the previous 15 days were also excluded. The modified Kirby-Bauer disc diffusion method was used for determining antimicrobial resistance against various antimicrobials.

\section{Results}

Out of 1,000 tested specimens, 530 (53\%) isolates were found to be culture-positive. E.coli was the most common species isolated from the cultures with a prevalence of $77.4 \%$, followed by Klebsiella (6.4\%), Enterobacter (6.0\%), Pseudomonas (3.8\%), Staphylococcus saprophyticus (3.4\%), Citrobacter (1.1\%), and Morganella ( $0.4 \%)$. Antimicrobial resistance against commonly used antimicrobials was found to be alarmingly high.

Received 07/30/2020 Review began $07 / 31 / 2020$ Review ended 07/31/2020 Published 08/14/2020

\section{() Copyright 2020}

Rizvi et al. This is an open access article distributed under the terms of the Creative Commons Attribution License CC-BY 4.0., which permits unrestricted use, distribution, and reproduction in any medium, provided the original author and source are credited.

\section{Conclusion}

E.coli was the most commonly isolated microorganism from the urine samples of UTI patients. Antimicrobial resistance against UTI-causing organisms is of great concern. The Surveillance of trends of antimicrobial susceptibility pattern for organisms causing UTIs is highly important. Antibiotics should be prescribed according to proper guidelines to prevent increasing antimicrobial resistance.

Categories: Urology, Infectious Disease

Keywords: urinary tract infections, antimicrobial resistance

\section{Introduction}

Urinary tract infections (UTIs) are one of the most commonly diagnosed diseases in outpatient departments (OPD) [1]. The selection of antibiotic therapy by a physician to treat UTI is based on the knowledge of prevalent microorganisms, recent updates about the antimicrobial susceptibility patterns, and the clinical status of the patient [2]. Studies have shown that E.coli is the most commonly isolated microorganism from UTI patients with a varying prevalence ranging from 26 to $55 \%$ [3,4].

There is conflicting data in the literature pertaining to the antimicrobial susceptibility patterns of UTIcausing organisms. A study conducted in Ethiopia showed a varying spectrum of antibiotic sensitivity pattern with $93.3 \%$ of the isolates being sensitive to gentamicin; however, less than $60 \%$ of the isolates were sensitive to chloramphenicol, nitrofurantoin, ciprofloxacin, trimethoprim-sulfamethoxazole (TMP-SMX), ceftriaxone, and nalidixic acid [5]. Another study showed a relatively lower susceptibility towards these antimicrobial agents, suggesting an increase in resistance towards these antibiotics [6]. The situation continues to worsen, especially in developing countries where the lack of appropriate surveillance and improper use of antibiotics contribute towards increased resistance in UTI-causing microorganisms $[5,6]$. 
Therefore, there is a need to conduct a study to determine the prevalence of most common agents associated with UTIs and their current antimicrobial resistance patterns, in order to formulate better antimicrobial therapies. Thus, our study aims to determine the frequency of agents causing UTI and their current antimicrobial resistance profiles. We believe this will contribute to the existing knowledge about antimicrobial resistance and ultimately improve treatment modalities for patients suffering from UTIs.

\section{Materials And Methods}

This descriptive cross-sectional study was conducted among patients presenting to the urology OPD of Benazir Bhutto Hospital, Rawalpindi, Pakistan from January 2017 till June 2017. Patients presenting to the OPD with typical clinical symptoms (burning micturition, fever, and pelvic pain) of UTI were included in the study. Patients with comorbid conditions including diabetes mellitus, renal pathologies, immunodeficiency disorders, malignancies, and congenital urogenital disorders were excluded based on their history. Patients receiving corticosteroid therapy or broad-spectrum antibiotics for the previous 15 days were also excluded from the study. The study was granted ethical approval by the Institutional Review Board of Rawalpindi Medical University. Consent was obtained from all participants and confidentially was maintained. Patients who did not give consent or refused to be a part of the study were also excluded.

Keeping in mind the exclusion criteria, the subjects were provided with a wide-mouthed standard-sized sterile container. They were advised to clean the area around urethra with water before collecting the sample, let the area dry, and collect the sample by catching it mid-stream with the container being held at 23 inches away. $50 \mu \mathrm{l}$ of urine was taken on a clean slide and a coverslip was placed on it. The slide was then viewed under a microscope. The presence of blood cells, epithelial cells, pus cells, or cast bodies was duly noted. The presence of 10 or more pus cells per high-power field was considered significant pyuria. The gram-staining technique was employed. Detection of at least one or more morphologically similar bacteria per oil immersion field was considered significant.

$10 \mu \mathrm{L}$ of the specimen was transferred to a MacConkey Agar plate using a calibrated loop method. The agar plates were incubated at $35-37^{\circ} \mathrm{C}$ for 24 hours for the identification of lactose-fermenting and non-lactosefermenting bacteria. A specimen was considered positive for UTI if a single organism was cultured at a concentration of 105 colony-forming units/ml. Cysteine lactose electrolyte deficient (CLED) medium was used for the identification and isolation of urinary pathogens.

The modified Kirby-Bauer disc diffusion method was used for determining the antimicrobial susceptibility. The colonies were placed on agar plates using a sterile inoculating wire loop. Antibiotic disks were placed using sterile forceps. The plates were left for one hour at room temperature to allow for the diffusion of antibiotics from the disks. The agar plates were again incubated for 24 hours at $37^{\circ} \mathrm{C}$. Antimicrobial susceptibility and resistance were tested against ampicillin, amoxicillin+clavulanic acid (AMC), gentamicin, amikacin, cefoperazone, ceftazidime, cefixime, ceftriaxone, cefepime, piperacillin+tazobactam (TZP), cefoperazone+sulbactam (CFP+SUL), carbapenem, fosfomycin, trimethoprim/sulfamethoxazole (SXT), ciprofloxacin, ofloxacin, levofloxacin, norfloxacin, and nitrofurantoin.

The frequencies and percentages of various microorganisms isolated from the urine samples were tabulated. The antimicrobial resistance profiles of the isolated organisms were expressed in percentages. The data were analyzed using SPSS Statistics version 23 (IBM, Armonk, NY).

\section{Results}

Out of 1,000 clinically suspected UTI patients, 530 (53\%) were found to be culture-positive, while 470 (47\%) were culture-negative. E.coli was the most frequently isolated microorganism, followed by Klebsiella spp., Enterobacter spp., Pseudomonas spp., Staphylococcus spp., Proteus spp., Citrobacter spp., and Morganella spp. The frequencies and percentages of different isolated microorganisms across gender are delineated in Table 1. 


\section{Cureus}

\begin{tabular}{|c|c|c|c|}
\hline Isolated microorganism & Total patients $(n=530)$ & Males $(n=160)$ & Females $(n=370)$ \\
\hline E.coli & $410(77.4 \%)$ & $116(72.5 \%)$ & $294(79.5 \%)$ \\
\hline Klebsiella spp. & $34(6.4 \%)$ & $10(6.2 \%)$ & $24(6.5 \%)$ \\
\hline Enterobacter spp. & $32(6 \%)$ & $4(2.5 \%)$ & $28(7.6 \%)$ \\
\hline Pseudomonas spp. & $20(3.8 \%)$ & $14(8.8 \%)$ & $6(1.6 \%)$ \\
\hline Staphylococcus spp. & $18(3.4 \%)$ & $10(6.2 \%)$ & $8(2.2 \%)$ \\
\hline Proteus spp. & $8(1.5 \%)$ & $4(2.5 \%)$ & $4(1.1 \%)$ \\
\hline Citrobacter spp. & $6(1.1 \%)$ & $2(1.2 \%)$ & $4(1.1 \%)$ \\
\hline Morganella spp. & $2(0.4 \%)$ & 0 & $2(0.5 \%)$ \\
\hline
\end{tabular}

TABLE 1: Frequencies and percentages of various isolated microorganisms from urinary samples

The isolated microorganisms had a varying spectrum of antimicrobial resistance against a plethora of antibiotics. This is elucidated in Table 2. 


\section{Cureus}

\begin{tabular}{|c|c|c|c|c|c|c|c|c|}
\hline $\begin{array}{l}\text { Antimicrobial } \\
\text { agent }\end{array}$ & $\begin{array}{l}\text { E. } \\
\text { coli }\end{array}$ & $\begin{array}{l}\text { Klebsiella } \\
\text { spp. }\end{array}$ & $\begin{array}{l}\text { Enterobacter } \\
\text { spp. }\end{array}$ & $\begin{array}{l}\text { Pseudomonas } \\
\text { spp. }\end{array}$ & $\begin{array}{l}\text { Staphylococcus } \\
\text { spp. }\end{array}$ & $\begin{array}{l}\text { Proteus } \\
\text { spp. }\end{array}$ & $\begin{array}{l}\text { Citrobacter } \\
\text { spp. }\end{array}$ & $\begin{array}{l}\text { Morganella } \\
\text { spp. }\end{array}$ \\
\hline Ampicillin & 87.3 & 100.0 & 50.0 & 0.0 & 100.0 & 100.0 & 100.0 & 100.0 \\
\hline AMC & 62.7 & 100.0 & 50.0 & 0.0 & 0.0 & 2.0 & 100.0 & 100.0 \\
\hline Gentamicin & 31.0 & 43.8 & 86.7 & 40.0 & 12.5 & 33.3 & 66.7 & 0.0 \\
\hline Amikacin & 1.0 & 25.0 & 0.0 & 20.0 & 0.0 & 33.3 & 0.0 & 0.0 \\
\hline Cefoperazone & 100.0 & 100.0 & 100.0 & 20.0 & 0.0 & 100.0 & 0.0 & 0.0 \\
\hline Ceftazidime & 100.0 & 100.0 & 100.0 & 22.2 & 0.0 & 50.0 & 0.0 & 0.0 \\
\hline Cefixime & 66.0 & 50.0 & 66.7 & 91.7 & 0.0 & 25.0 & 0.0 & 0.0 \\
\hline Ceftriaxone & 63.7 & 47.1 & 66.7 & 100.0 & 0.0 & 25.0 & 0.0 & 0.0 \\
\hline Cefepime & 63.2 & 43.8 & 66.7 & 22.2 & 0.0 & 0.0 & 0.0 & 0.0 \\
\hline TZP & 11.3 & 17.6 & 33.3 & 22.2 & 0.0 & 0.0 & 66.7 & 0.0 \\
\hline CFP+SUL & 10.3 & 29.4 & 33.3 & 22.2 & 0.0 & 0.0 & 66.7 & 0.0 \\
\hline carbapenem & 2.5 & 5.9 & 16.7 & 22.2 & 0.0 & 33.3 & 0.0 & 0.0 \\
\hline Fosfomycin & 2.0 & 0.0 & 20.0 & 0.0 & 33.3 & 100.0 & 33.3 & 100.0 \\
\hline SXT & 71.9 & 52.9 & 66.7 & 33.3 & 33.3 & 25.0 & 66.7 & 0.0 \\
\hline Ciprofloxacin & 75.4 & 47.1 & 87.5 & 60.0 & 0.0 & 0.0 & 33.3 & 0.0 \\
\hline Ofloxacin & 100.0 & 100.0 & 100.0 & 70.0 & 0.0 & 0.0 & 0.0 & 0.0 \\
\hline Levofloxacin & 93.3 & 88.9 & 100.0 & 77.8 & 75.0 & 50.0 & 0.0 & 0.0 \\
\hline Norfloxacin & 75.4 & 40.0 & 0.0 & 70.0 & 42.9 & 33.3 & 66.7 & 0.0 \\
\hline Nitrofurantoin & 10.7 & 70.6 & 0.0 & 66.7 & 0.0 & 100.0 & 33.3 & 100.0 \\
\hline
\end{tabular}

TABLE 2: Antimicrobial resistance profile of various UTI-causing microorganisms against commonly used antibiotics

The values are expressed in percentages

UTI: urinary tract infection; AMC: amoxicillin+clavulanic acid; TZP: piperacillin+tazobactam; CFP+SUL: cefoperazone+sulbactam; SXT: trimethoprim/sulfamethoxazole

\section{Discussion}

UTIs are a very common problem and represent a major burden on healthcare systems, especially in developing countries [6]. Our study showed a plethora of organisms causing UTIs with varying patterns of resistance against many broad-spectrum antibiotics. UTIs are becoming difficult to treat owing to increasing resistance and intensifying global disease burden [7,8]. The emergence of resistance against broad-spectrum antibiotic agents such as extended-spectrum beta-lactams, fluoroquinolones, and carbapenem is causing problems worldwide [9].

E.coli was the most commonly isolated pathogen from our tested urine samples with a high prevalence of $77.4 \%$. This overwhelming presence of E.coli was also observed in another study with $53.6 \%$ samples being culture-positive for E.coli, 14.6\% for Proteus, 13.9\% for Klebsiella, 4.5\% for Enterococcus, and $4.1 \%$ for Staphylococcus [10]. In another study, it was observed that $60 \%$ of isolates from urinary samples were of E.coli, 12\% of Klebsiella, and 8\% of Enterococcus [11]. Similarly, a study conducted in India indicated an alarmingly high prevalence of $69.8 \%$ for E.coli, followed by Klebsiella (7.9\%), Staphylococcus (4.8\%), and $1.6 \%$ for methicillin-resistant Staphylococcus aureus (MRSA) [12]. The results obtained from these studies are comparable to our observations, indicating very similar trends in the prevalence of various causative organisms for UTI.

The antimicrobial resistance profile of E.coli in our study showed that more than $75 \%$ of the strains were resistant to fluoroquinolones; however, the resistance profile towards more broad-spectrum antibiotics 
including nitrofurantoin, fosfomycin, and carbapenem ranged between 2-10.7\%. Another study indicated an alarming resistance rate of E.coli towards broad-spectrum fluoroquinolones with $60 \%$ of strains being resistant to them [4]. The resistance patterns of E.coli in our study were also similar to another study that observed an $81.8 \%$ resistant strains of E.coli to ampicillin, $54.5 \%$ to cefepime, $2.3-6.8 \%$ to carbapenem, $36.4 \%$ to gentamicin, $9.1 \%$ to amikacin, up to $66 \%$ for fluoroquinolones, and $15.9 \%$ to nitrofurantoin [12]. Another study reported that $40.5 \%$ strains of Klebsiella were resistant to gentamicin, $5.4 \%$ to carbapenem, $78.4 \%$ to levofloxacin, and $83.8 \%$ to nitrofurantoin, while according to our observations, resistance rates were $43.8 \%, 5.9 \%, 88.9 \%$, and $70.6 \%$, respectively [13]. Another study showed a similar resistance profile of E.coli against ampicillin with $69.5 \%$ of strains being resistant to it [14]. A study conducted in the United States indicated that the resistance among isolates of E.coli from urinary samples was lowest for nitrofurantoin $(<1 \%)[15]$. This finding was quite in contrast with our observation where the resistance profile of E.coli against nitrofurantoin was found to be $10.7 \%$. However, another study reported the resistance rate of E.coli against nitrofurantoin to be around 15\%, which is consistent with our findings [16].

The increasing resistance of E.coli towards broad-spectrum antibiotics like nitrofurantoin is of great concern and demands the need for appropriate antibiotic use according to the recommended guidelines. As there is no strict adherence to antibiotic stewardship, the clinicians tend to prescribe broad-spectrum antibiotics that contribute to increased antimicrobial resistance $[17,18]$. Researchers have recommended conducting quality improvement programs to ensure appropriate antibiotic use according to the proposed guidelines, especially in a developing country like ours. The increased resistance of common microorganisms causing UTI is of huge concern for the clinicians as providing broad-spectrum alternatives can predispose the patients towards their horrendous adverse effects. Adequate control on antibiotic prescription according to proper guidelines will aid in the delivery of top-quality patient care and prevent the development of multidrug-resistant organisms.

This article has been posted on a preprint server (bioRxiv) [19]. The preprint version is not pending full publication elsewhere.

\section{Conclusions}

E.coli is the most commonly isolated pathogen from urine samples of clinically suspected UTI patients. Antimicrobial resistance in UTI-causing agents against commonly used antimicrobials is extremely frightening. The alarming antimicrobial resistance profile of UTI-causing organisms strongly indicates the need to establish proper guidelines for the use of antibiotics in the treatment of UTI patients. We hope this study opens up new horizons for advanced quality improvement projects and further research on appropriate antibiotic prescription and usage.

\section{Additional Information \\ Disclosures}

Human subjects: Consent was obtained by all participants in this study. Institutional Review Board, Rawalpindi Medical University issued approval RSRS-2017-SS-7B313. Ethical approval was granted by the Institutional Research Board of Rawalpindi Medical University. Animal subjects: All authors have confirmed that this study did not involve animal subjects or tissue. Conflicts of interest: In compliance with the ICMJE uniform disclosure form, all authors declare the following: Payment/services info: All authors have declared that no financial support was received from any organization for the submitted work. Financial relationships: All authors have declared that they have no financial relationships at present or within the previous three years with any organizations that might have an interest in the submitted work. Other relationships: All authors have declared that there are no other relationships or activities that could appear to have influenced the submitted work.

\section{References}

1. Gales AC, Jones RN, Gordon KA, et al.: Activity and spectrum of 22 antimicrobial agents tested against urinary tract infection pathogens in hospitalized patients in Latin America: report from the second year of the SENTRY antimicrobial surveillance program (1998). J Antimicrob Chemother. 2000, 45:295-303. 10.1093/jac/45.3.295

2. Haller M, Brandis M, Berner R: Antibiotic resistance of urinary tract pathogens and rationale for empirical intravenous therapy. Pediatr Nephrol. 2004, 19:982-986. 10.1007/s00467-004-1528-7

3. Kuzdan C, Soysal A, Culha G, Altinkanat G, Soyletir G, Bakir M: Three-year study of health care-associated infections in a Turkish pediatric ward. J Infect Dev Ctries. 2014, 8:1415-1420. 10.3855/jidc.3931

4. Gupta S, Kapur S, Padmavathi D: Comparative prevalence of antimicrobial resistance in communityacquired urinary tract infection cases from representative States of northern and southern India. J Clin Diagn Res. 2014, 8:DC09-12. 10.7860/JCDR/2014/9349.4889

5. Seifu WD, Gebissa AD: Prevalence and antibiotic susceptibility of uropathogens from cases of urinary tract infections (UTI) in Shashemene referral hospital, Ethiopia. BMC Infect Dis. 2018, 18:30. 10.1186/s12879017-2911-x

6. Shaifali I, Gupta U, Mahmood SE, Ahmed J: Antibiotic susceptibility patterns of urinary pathogens in female outpatients. N Am J Med Sci. 2012, 4:163-169. 10.4103/1947-2714.94940

7. Pouladfar G, Basiratnia M, Anvarinejad M, Abbasi P, Amirmoezi F, Zare S: The antibiotic susceptibility 
patterns of uropathogens among children with urinary tract infection in Shiraz. Medicine (Baltimore). 2017, 96:e7834. 10.1097/MD.0000000000007834

8. Foxman B: Urinary tract infection syndromes: occurrence, recurrence, bacteriology, risk factors, and disease burden. Infect Dis Clin North Am. 2014, 28:1-13. 10.1016/j.idc.2013.09.003

9. Chen YH, Ko WC, Hsueh PR: Emerging resistance problems and future perspectives in pharmacotherapy for complicated urinary tract infections. Expert Opin Pharmacother. 2013, 14:587-596. 10.1517/14656566.2013.778827

10. Das R, Perrelli E, Towle V, Van Ness PH, Juthani-Mehta M: Antimicrobial susceptibility of bacteria isolated from urine samples obtained from nursing home residents. Infect Control Hosp Epidemiol. 2009, 30:11161119. 10.1086/647981

11. Sundvall PD, Ulleryd P, Gunnarsson RK: Urine culture doubtful in determining etiology of diffuse symptoms among elderly individuals: a cross-sectional study of 32 nursing homes. BMC Fam Pract. 2011, 12:36. 10.1186/1471-2296-12-36

12. George CE, Norman G, Ramana GV, Mukherjee D, Rao T: Treatment of uncomplicated symptomatic urinary tract infections: resistance patterns and misuse of antibiotics. J Family Med Prim Care. 2015, 4:416-421. 10.4103/2249-4863.161342

13. Sugianli AK, Ginting F, Kusumawati RL, et al.: Antimicrobial resistance in uropathogens and appropriateness of empirical treatment: a population-based surveillance study in Indonesia. J Antimicrob Chemother. 2017, 72:1469-1477. 10.1093/jac/dkw578

14. Ilić T, Gračan S, Arapović A, Capkun V, Subat-Dežulović M, Saraga M: Changes in bacterial resistance patterns in children with urinary tract infections on antimicrobial prophylaxis at University Hospital in Split. Med Sci Monit. 2011, 17:CR355-361. 10.12659/msm.881845

15. Edlin RS, Shapiro DJ, Hersh AL, Copp HL: Antibiotic resistance patterns of outpatient pediatric urinary tract infections. J Urol. 2013, 190:222-227. 10.1016/j.juro.2013.01.069

16. Gupta K, Hooton TM, Naber KG, et al.: International clinical practice guidelines for the treatment of acute uncomplicated cystitis and pyelonephritis in women: a 2010 update by the Infectious Diseases Society of America and the European Society for Microbiology and Infectious Diseases. Clin Infect Dis. 2011, 52:e103120. 10.1093/cid/ciq257

17. Satti MZ, Hamza M, Sajid Z, Asif O, Ahmed H, Zaidi SM, Irshad U: Compliance rate of surgical antimicrobial prophylaxis and its association with knowledge of guidelines among surgical residents in a tertiary care public hospital of a developing country. Cureus. 2019, 11:e4776. 10.7759/cureus.4776

18. Zaidi SM, Kaneez M, Almas T, et al.: Gauging the risk factors for asymptomatic bacteriuria in type-2 diabetic women: a case-control study. Cureus. 2020, 12:e9069. 10.7759/cureus.9069

19. Rizvi ZA, Jamal AM, Malik AH, Ullah N, Arshad D: Anti-microbial resistance in agents causing urinary tract infections [PREPRINT]. bioRxiv. 2018, 10.1101/316869 